\title{
Aspectos radiográficos da doença valvar crônica
}

\author{
Radiographic aspects of chronic valvular disease \\ Elaine Cristina Soares ${ }^{1}$ Maria Helena Matiko Akao Larsson ${ }^{2}$ \\ Ana Carolina Brandão de Campos Fonseca Pinto ${ }^{3}$
}

\section{RESUMO}

\begin{abstract}
O exame radiográfico é um dos métodos mais utilizados para o diagnóstico da doença valvar crônica, seja para avaliação da silhueta cardíaca seja para avaliação de campos pulmonares, que podem apresentar anormalidades decorrentes da insuficiência cardíaca. Um dos métodos de avaliação da silhueta cardíaca é o sistema "vertebral heart size", proposto por BUCHANAN e BÜCHELER (1995). Setenta cães portadores de sopro à auscultação, sintomáticos ou não, foram caracterizados quanto à severidade dos sinais clínicos e à intensidade do sopro presente. As radiografias de todos os animais foram analisadas pelo método de mensuração "VHS" . Os dados clínicos e radiográficos foram comparados entre si, verificando-se que, quanto mais severos os sinais clínicos, maior a probabilidade de se encontrarem alterações radiográficas indicativas de cardiomegalia. Observou-se, ainda, que, quanto maior a intensidade do sopro, mais freqüentes e severas são as alterações radiográficas.
\end{abstract}

Palavras-chave: valvas cardíacas, insuficiência cardíaca, cães, coração, radiografia.

\section{ABSTRACT}

The radiographic examination is one of the most applied diagnostic method in chronic valvular disease, evaluating the cardiac silhouette and/or lung abnormalities. The radiographic cardiac image can be measured by the "vertebral heart size" system, proposed by BUCHANAN and BÜCHELER (1995). Seventy dogs who presented murmur on auscultation, symptomatic or not, were studied, and the radiographic examination of them were evaluated by the "VHS" method. Clinical and radiographic findings were compared, indicating that the worse the severity of symptoms the higher the probability of finding cardiomegaly in radiographies. Besides, the higher the murmur intensity the more often the radiographic signs of cardiomegaly were observed.

Key words: cardiac valves, cardiac insufficiency, dogs, heart, radiography.

\section{INTRODUÇÃO}

O exame radiográfico é um dos métodos diagnósticos mais utilizados para avaliar a silhueta cardíaca e detectar conseqüências decorrentes da fibrose valvar crônica. A imagem radiográfica da silhueta cardíaca representa a composição de diferentes estruturas (FAGIN, 1998), o que torna imprescindível o conhecimento da anatomia radiográfica, a fim de que se torne possível a identificação dos diferentes componentes da imagem da silhueta cardíaca.

O método mais freqüentemente utilizado para a avaliação da silhueta cardíaca é o empírico, no qual o profissional faz uso de seus conhecimentos sobre anatomia radiográfica, a fim de detectar alterações de forma e tamanho de partes específicas do coração. As vantagens desse método são basicamente a conveniência e rapidez (SUTER, 1984), porém deve ser adotado apenas por radiologistas experientes, que conheçam as variações normais que a imagem radiográfica do coração pode apresentar.

Visando diminuir a subjetividade do método empírico, vários métodos de mensuração foram

${ }^{1}$ Mestre em Clínica Veterinária pela Faculdade de Medicina Veterinária e Zootecnia (FMVZ), Universidade de São Paulo (USP). ${ }^{2}$ Professor Associado, Departamento de Clínica Médica, FMVZ-USP. Avenida Ceci, 543, Planalto Paulista, 04064-000, São Paulo, SP. E-mail: akaolar@usp.br Autor para correspondência.

${ }^{3}$ Professor Assistente, Departamento de Cirurgia, FMVZ-USP 
propostos. FONSECA (1999), estudando os métodos de mensuração propostos por Schulze e Nöldner, Hamlim e Buchanan e Bücheler, concluiu que aquele descrito por BUCHANAN \& BÜCHELER (1995) é o mais indicado para ser utilizado como método de triagem das cardiomegalias. Os referidos autores propuseram um sistema de mensuração denominado "Vertebral Heart Size" (VHS), que compara o comprimento do eixo longo e do eixo curto do coração, com o número de vértebras torácicas correspondentes, iniciando a partir de T4, e estimando-se o valor desses eixos numa escala vertebral com aproximação de 0,1 vértebra. Os valores normais de "VHS" determinados por BUCHANAN \& BÜCHELER (1995) em projeções laterais, foram menores ou iguais a 10,5 vértebras em $98 \%$ das radiografias dos cães estudados, valor esse considerado limite (superior) para a silhueta cardíaca da maioria das raças caninas. Para cães de tórax curto, como os Schnauzer miniaturas, considera-se como normal um valor de VHS até 11 vértebras (v). Por outro lado, para aqueles cães que possuem tórax longo, como, por exemplo, os Dachshunds, considera-se como normal o valor limite de 9,5 vértebras (v).

FONSECA (1999), utilizando esse método proposto, para cães das raças Cocker Spaniel, Boxer e Doberman, obteve, respectivamente, valores médios de VHS iguais a $11,05 \mathrm{v}, 11,51 \mathrm{v}$, e $10,33 \mathrm{v}$, nas mensurações realizadas na projeção lateral esquerda direita. Segundo o autor, houve uma tendência em superestimar o tamanho do lado direito do coração, quando se fez uso da avaliação empírica, principalmente nos animais da raça Cocker Spaniel.

Embora o método VHS seja de fácil aplicação, mesmo para profissionais inexperientes, não permite a identificação de quais câmaras cardíacas estão aumentadas, uma vez que o eixo curto inclui tanto câmaras direitas quanto esquerdas.

Algumas desvantagens da utilização do sistema "Vertebral Heart Size" são a grande variação de conformação torácica entre as diferentes raças, a utilização de apenas uma projeção radiográfica, radiografias obtidas em momentos diferentes do ciclo respiratório (inspiração ou expiração), variações no eixo do coração, mudanças na forma da caixa torácica, causadas por doenças congênitas ou adquiridas, e alterações intra ou extratorácicas que levem a mudanças nas proporções cardiotorácicas (BUCHANAN \& BÜCHELER, 1995; SUTER, 1984; TOOMBS \& OGBURN, 1985). Apesar destes aspectos, o método ainda é considerado válido para ser utilizado por profissionais inexperientes.

As radiografias de indivíduos portadores de insuficiência valvar podem não apresentar anormalidades na silhueta cardíaca, quando a regurgitação mitral for discreta. Em casos de regurgitação moderada a severa, pode-se observar aumento no tamanho do átrio e ventrículo esquerdos (HURST et al., 1993). BONAGURA (1997) refere que a severidade da regurgitação mitral geralmente corresponde ao grau de dilatação atrial, observado radiograficamente.

$\mathrm{O}$ aspecto do parênquima e dos vasos pulmonares também pode ser analisado por meio das radiografias, desempenhando assim um papel importante no diagnóstico e prognóstico da condição mórbida. Nos casos de insuficiência cardíaca esquerda, pode-se observar hipervascularização pulmonar, com congestão venosa e infiltrado intersticial e alveolar. Já a insuficiência cardíaca direita está relacionada à distensão da veia cava caudal, hepatomegalia, ascite e, às vezes, hidrotórax (DARKE et al, 1996).

Particularmente nos casos de doença valvar mitral crônica, observa-se a compressão do brônquio principal esquerdo pelo átrio esquerdo dilatado, o que geralmente está associado à tosse (BONAGURA, 1997).

Procurou-se, neste estudo, analisar os aspectos radiográficos referentes à doença valvar crônica, correlacionando-os com os dados clínicos, como classe funcional da insuficiência cardíaca e intensidade de sopro.

\section{MATERIAL E MÉTODOS}

Foram estudados 70 cães sem definição racial e de raças diversas, machos e fêmeas, de idade variável e não tratados, os quais foram encaminhados ao Serviço de Cardiologia do Hospital Veterinário (HOVET) da Faculdade de Medicina Veterinária e Zootecnia da Universidade de São Paulo, por apresentarem sinais relacionados à insuficiência cardíaca ou, simplesmente, por apresentarem sopro à auscultação, independentemente da presença de sinais clínicos.

\footnotetext{
Anamnese

A anamnese foi realizada inquirindo-se a respeito de sinais referentes à insuficiência cardíaca, como tosse, cansaço fácil, dispnéia, cianose, edema de membros e ascite. Esses dados, adicionados àqueles obtidos por meio de exames físico e complementares, permitiu a inclusão dos animais em uma das classe funcionais da insuficiência cardíaca, segundo critério descrito pelo International Small Animal Cardiac Health Council” (ISACHC).
} 


\section{Exame físico}

O exame físico constituiu-se no exame do tórax por meio de inspeção, palpação, percussão dígitodigital e auscultação indireta com o animal em estação, utilizando-se para isto um estetoscópio marca Littmann ${ }^{\circledR}$, modelo Cardiology II SE. A auscultação cardíaca possibilitou a detecção de sopros, que então foram classificados segundo o foco e a intensidade, esta última de acordo com a escala de Friedman (DARKE et al, 1996), variando de 1 a 6.

\section{Radiografias}

Os exames radiográficos da cavidade torácica foram realizados em aparelhos de radiodiagnóstico, marca CGR®, de $300 \mathrm{~mA}$ e $125 \mathrm{kV}$, modelo $\mathrm{CH} 3000$, equipado com quatro retificadores de silicone, mesa radiológica com grade e sistema "Potter-bucky" recipromático tipo "par speed", ampolas de raios $\mathrm{X}$ de ânodo giratório. Os filmes radiográficos utilizados - marca $\mathrm{RPX}-\mathrm{OMAT}^{(1)}$ - eram de tamanhos $18 \times 24 \mathrm{~cm}, 24 \times 30 \mathrm{~cm}$ e $30 \times 40 \mathrm{~cm}$, de acordo com o porte do animal, colocados em chassi metálico, portando telas intensificadoras CRONEX HI plus $^{(2)}$. Os filmes foram revelados e fixados em processadora automática RP-OMAT Processor (3), após a identificação luminosa apropriada.

Os animais foram posicionados em decúbito lateral direito, no caso de projeção lateral esquerda-direita e, em decúbito lateral esquerdo, no caso de projeção lateral direita-esquerda, em decúbito esternal, no caso de projeção dorso-ventral e, em decúbito dorsal, no caso de projeção ventro-dorsal. As técnicas radiográficas utilizadas foram as que relacionam a quilovoltagem e a miliamperagem-segundo a espessura da região a ser radiografada.
A análise das radiografias foi realizada pelo método de mensuração "Vertebral Heart Size" descrito por BUCHANAN \& BÜCHELER (1995) (Figura 1). Para tanto, em radiografia lateral esquerda-

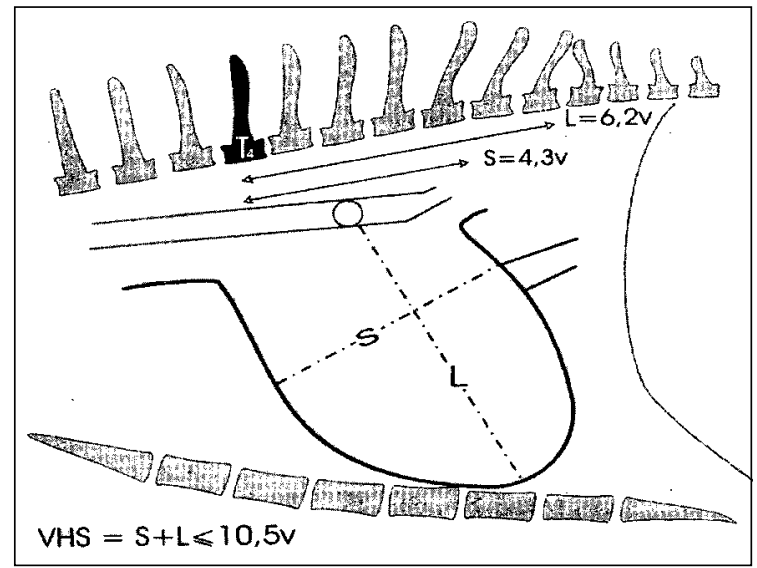

Figura 1 - Método de mensuração proposto por BUCHANAN E BÜCHELER (1995) na projeção látero-lateral. L= eixo maior do coração (distância da margem ventral do brönquio principal esquerdo ao ponto mais distante do contorno ventral do ápice cardíaco): $\mathrm{S}=$ eixo menor do coração (linha perpendicular ao eixo maior na altura do terço central do coração): $\mathrm{T}_{4}=$ Quarta vértebra torácica. As medidas de L e S são transportadas para a coluna vertebral torácica, colocando-se uma das pontas do compasso junto à margem cranial da quarta vértebra torácica $\mathrm{T}_{4}$, e estimando-se o valor desses eixos numa escala vertebral com aproximação de 0,1 vértebra: VHS = " vertebral heart size" ou "tamanho do coração em ralação à unidade de vértebra torácica". Para projeções laterais: VHS $=10,5$ vértebras $(\mathrm{v})$ - limite superior para um tamanho normal de silhueta cardíaca na maioria das raças: VHS $=11$ vértebras $(v)-$ cães com tórax curto como os Schnauzer miniaturas, VHS = 9,5 vértebras (v) - cães com tórax longo, como os Dachshunds.

Tabela 1 - Distribuição dos casos de fibrose valvar crônica segundo a classe funcional da insuficiência cardíaca e os achados radiográficos, atendidos no Serviço de Cardiologia-HOVET-USP, São Paulo-JUL. 1999/ JAN. 2001.

\begin{tabular}{|c|c|c|c|c|}
\hline $\mathrm{RX}^{(2)}$ & $\mathrm{CF}^{(1)}$ & Cardiomegalia & Sem alterações & TOTAL \\
\hline \multirow{2}{*}{ I } & IA $(n=8)$ & $25 \%$ & $75 \%$ & $100 \%$ \\
\hline & IB $(n=8)$ & $25 \%$ & $75 \%$ & $100 \%$ \\
\hline II & $(n=42)$ & $52,4 \%$ & $47,6 \%$ & $100 \%$ \\
\hline \multirow{2}{*}{ III } & IIIA $(n=8)$ & $100 \%$ & - & $100 \%$ \\
\hline & IIIB $(n=4)$ & $100 \%$ & - & $100 \%$ \\
\hline
\end{tabular}

(1) classe funcional da insuficiência cardíaca; IA (animal assintomático, com sinais de cardiopatia como sopros, arritmias ou aumento de câmaras à radiografia de tórax ou ecocardiograma, mas sem hipertrofia por sobrecarga pressórica ou volumétrica); IB (animal assintomático, com sinais de cardiopatia e com hipertrofia por sobrecarga pressórica ou volumétrica); II (insuficiência cardíaca leve a moderada); IIIA (insuficiência cardíaca avançada, mas possível o tratamento em casa); IIIB (nsuficiência cardíaca avançada, sendo necessária a hospitalização).

(2) achados radiográficos, considerando a presença ou não de cardiomegalia, de acordo com os valores obtidos pelo método "Vertebral Heart Size" 
direita, considerou-se como eixo longo do coração a distância da margem ventral do brônquio principal esquerdo ao contorno ventral mais distante do ápice cardíaco, que reflete o tamanho combinado do átrio e ventrículo esquerdos. Esta medida foi transportada para a coluna vertebral torácica, colocando-se uma das pontas de um compasso junto à margem cranial da quarta vértebra torácica, e estimando-se o valor deste eixo numa escala vertebral com aproximação de 0,1 vértebra. Para a obtenção do eixo curto do coração, posicionou-se o compasso junto ao terço superior do coração, de forma que se obtivesse uma linha perpendicular ao eixo longo, e realizou-se o mesmo procedimento para a obtenção do seu tamanho, transformado em unidades de vértebra torácica. As medidas do eixo longo e curto foram somadas, a fim de que se obtivesse uma expressão do tamanho do coração, com relação ao indicador vertebral do comprimento do corpo. O tamanho total do coração foi expresso em unidades de vértebras, com aproximação de 0,1 vértebra, ao qual se designou "VHS".

\section{RESULTADOS}

Os achados radiográficos quanto à presença ou não de aumento da silhueta cardíaca, considerando-se os valores de VHS, foram comparados com a classe funcional (ISACHC) e intensidade do sopro, dispostos em forma de tabelas (Tabelas 1 e 2 respectivamente). Consideraram-se normais os valores de VHS iguais ou menores que 10,5v (BUCHANAN \& BÜCHELER, 1995).

Analisando o quadro 1, pode-se observar que a maioria dos animais assintomáticos, em classe funcional I, não apresentaram aumento da silhueta cardíaca ao RX. Por outro lado, cardiomegalia foi caracterizada radiograficamente em todos os cães com doença avançada (classe funcional III) (Figura 2). Já os incluídos em classe funcional II distribuíram-se equilibradamente, isto é, aproximadamente metade dos animais apresentando alterações radiográficas e o restante demonstrando silhueta cardíaca de tamanho normal, de acordo com os valores obtidos pelo método VHS.

Outra associação, disposta no quadro 2 , foi realizada entre os achados radiográficos e a intensidade de sopro presente à auscultação. O quadro 2 mostra que a maior parte dos animais portadores de sopro de intensidade baixa (graus 1 e 2), ou moderada (grau 3) não apresentam, radiograficamente, aumento global da silhueta cardíaca.

Dos 19 casos portadores de sopro de alta intensidade, acompanhado de frêmito pré-cordial (graus 5 e 6), apenas 1 , ou 6,7\%, não apresentava aumento global da silhueta cardíaca. Aqueles cujo sopro foi classificado como de intensidade moderada a alta (grau 4), porém sem frêmito pré-cordial, demonstrou uma distribuição equilibrada entre os que apresentou cardiomegalia e os que não revelaram alteração radiográfica relacionada ao tamanho da imagem cardíaca.

\section{DISCUSSÃO}

Pacientes com fibrose valvar crônica, porém assintomáticos (classe I), não revelaram, na maioria das vezes, alterações radiográficas. Por outro lado, todos aqueles com sinais severos, descompensados, apresentaram cardiomegalia. Não se observou nenhuma associação entre a severidade da doença e os achados radiográficos nos animais portadores de sinais leves a moderados (CF II).

Dados semelhantes foram descritos por AMBERGER et al. (1995) que, utilizando as normas de classificação da "New York Heart Association", mostraram que o número de radiografias normais decresce com o aumento da classe funcional da insuficiência cardíaca. Esses autores encontraram, porém, uma porcentagem menor de radiografias normais nos casos de insuficiência cardíaca leve (CF I e CF II) e moderada (CF III). Dos animais em classe funcional IV, ou seja, insuficiência cardíaca avançada, nenhum apresentou radiografias normais. No entanto, deve-se ressaltar que o método de avaliação da silhueta cardíaca utilizados por este autor foi a análise subjetiva, e isto pode explicar o menor número de radiografias normais encontradas por ele nos casos de CF I, II e III, uma vez que, como já descrito, existe uma tendência em superestimar o tamanho da silhueta

Quadro 2 - Distribuição dos casos de fibrose valvar crônica, segundo a intensidade de sopro e achados radiográficos, atendidos no Serviço de CardiologiaHOVET-USP, São Paulo-JUL 1999./JAN.2001.

\begin{tabular}{|c|c|c|c|c|}
\hline IS $^{(1)}$ & $\mathrm{RX}^{(2)}$ & Cardiomegalia & Sem alterações & TOTAL \\
\hline \multicolumn{2}{|c|}{ Grau $1(n=3)$} & - & $100 \%$ & $100 \%$ \\
\hline \multicolumn{2}{|c|}{ Grau $2(n=3)$} & $25 \%$ & $75 \%$ & $100 \%$ \\
\hline \multicolumn{2}{|c|}{ Grau $3(n=17)$} & $35,3 \%$ & $64,7 \%$ & $100 \%$ \\
\hline \multicolumn{2}{|c|}{ Grau $4(n=28)$} & $46,4 \%$ & $53,6 \%$ & $100 \%$ \\
\hline \multicolumn{2}{|c|}{ Grau 5 (n=15) } & $93,3 \%$ & $6,7 \%$ & $100 \%$ \\
\hline \multicolumn{2}{|c|}{ Grau $6(n=4)$} & $100 \%$ & - & $100 \%$ \\
\hline
\end{tabular}

(1) intensidade do sopro presente à auscultação, segundo a escala de 1 a 6 de Friedman (DARKE et al, 1996).

(2) achados radiográficos, considerando a presença ou não de cardiomegalia, de acordo com os valores obtidos pelo método "Vertebral Heart Size" 


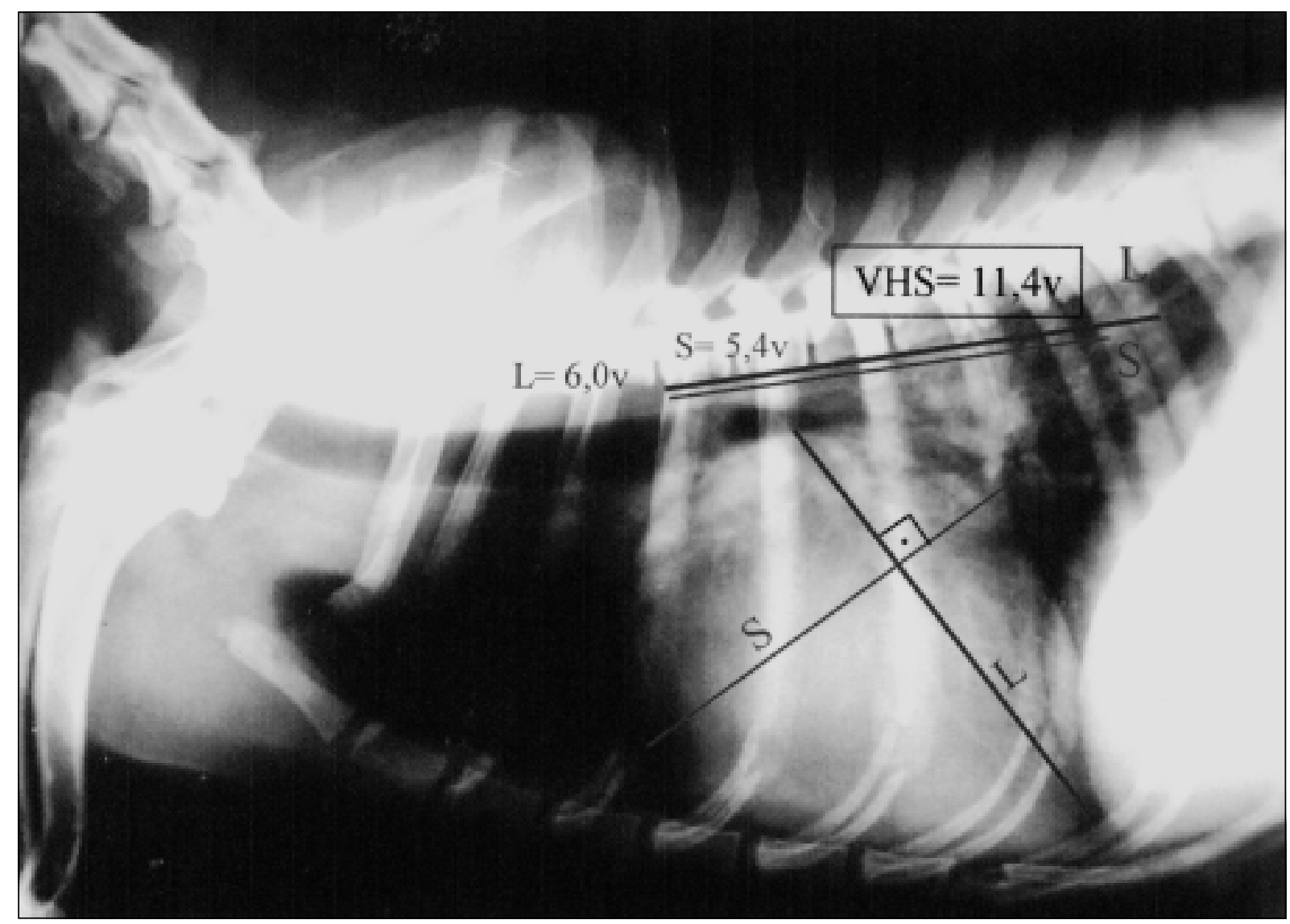

Figura 2 - Aplicação do método de VHS em figura de radiografia em projeção lateral esquerda-direita de toráx em cão portador de fibrose valvar crônica. Este animal apresentava-se em classe funcional IIIA. Observa-se valor de VHS acima dos valores normais.

cardíaca quando se faz uso da avaliação subjetiva.

Analisando-se a tabela 2, que compara a intensidade do sopro, presente à auscultação, e os achados radiográficos, verifica-se que a maioria dos cães portadores de sopro de intensidade baixa a moderada (graus 1, 2 e 3) não apresentaram aumento da silhueta cardíaca.

Aproximadamente $50 \%$ dos animais cuja ausculta permitiu a detecção de um sopro de intensidade moderada a alta, sem frêmito pré-cordial (grau 4), não apresentaram alterações radiográficas, e a mesma porcentagem demonstrou aumento global da silhueta cardíaca. Apenas um dos 19 cães portadores de sopro de intensidade alta, com frêmito pré-cordial (graus 5 e 6), não apresentou alteração radiográfica relacionada ao tamanho do coração.

Estes achados corroboram com os de BOUSFIELD \& BOWDEN (1972), que, estudando insuficiência mitral induzida cirurgicamente, observaram uma associação entre a presença de cardiomegalia e a intensidade do sopro, ou seja, animais portadores de sopros de intensidade maior apresentavam aumento global da silhueta cardíaca com maior frequência que aqueles portadores de sopro de baixa intensidade. Devese ressaltar, porém, que a incompetência valvar induzida cirurgicamente difere da naturalmente adquirida, principalmente no que concerne ao gradiente e velocidade do fluxo entre átrio e ventrículo, responsáveis em parte pela intensidade do sopro presente à auscultação. Não foram encontrados, contudo, estudos que comparam a intensidade do sopro com o tamanho da silhueta cardíaca, particularmente avaliado por meio do VHS.

\section{CONCLUSÕES}

Os resultados obtidos, por meio da metodologia utilizada, permitem dizer que:

As alterações radiográficas intensificam-se de forma crescente com a classe funcional da insuficiência cardíaca;

Quanto maior a intensidade do sopro, detectado à auscultação, mais freqüentes e severas 
são as alterações radiográficas indicativas de aumento global do coração.

\section{REFERÊNCIAS BIBLIOGRÁFICAS}

AMBERGER, C.; GLARDON, O.; LOMBARD, C.W. Validité des examens complementaires dans l'evaluation de l'insuffisance cardiaque par endocardiose mitrale. Ëtude a partir de 106 cas. Pratique Medicale and Chirurgicale de l'animal de Compagnie, v.30, n.6, p.659-670, 1995.

BONAGURA, J.D. Clinical evaluation of the cardiovascular system. In: ETTINGER/BONAGURA e os recentes avanços da cardiologia veterinária. São Paulo : Sociedade Brasileira de Cardiologia Veterinária, 1997. p.1-20 (apostila).

BOUSFIELD, W.E.D.; BOWDEN, L.R. Observations on surgically induced insufficiency of the canine mitral valve. British Veterinary Journal, v.128, n.11, p.567-577, 1972.

BUCHANAN, J.W.; BÜCHELER, J. Vertebral scale system to measure canine heart size in radiographs. Journal of the American Veterinary Medical Association, v.206, n.2, p.194-199, 1995.
DARKE, P.G.G.; BONAGURA,J.D.; KELLY, D.F. Color atlas of veterinary cardiology. London : Mosby-wolfe, 1996. $186 \mathrm{p}$.

FAGIN, B.D. The cardiac silhouette: centerpiece of the thoracic radiograph. Veterinary Medicine, v. 83, n.10, p.1033-1044, 1988.

FONSECA, A.C.B.C. Estudo dos métodos radiográficos de avaliação da silhueta cardíaca em cães clinicamente normais. 1999. 73f. Dissertação (Mestrado em clínica veterinária) - Faculdade de Medicina Veterinária e Zootecnia da Universidade de São Paulo.

HURST, J.W. et al. Doença cardíaca valvar. In: HURST, J.W. et al. Atlas do coração. São Paulo : Manole, 1993. p.4.14.60 .

SUTER, P.F. Cardiac diseases. In: SUTER, P.F. Thoracic radiography: a text atlas of thoracic diseases of the dog and cat. Switzerland : Peter F. Suter, 1984. Cap.10. p.365-367.

TOOMBS, J.P.; OGBURN, P.N. Evaluating canine cardiovascular silhouettes: radiographic methods and normal radiographic anatomy. The Compendium on Continuing Education in practicing veterinarian, v.7, n.7, p.579$587,1985$. 